\title{
CORRECTION OPEN
}

\section{Correction: Alpl prevents bone ageing sensitivity by specifically regulating senescence and differentiation in mesenchymal stem cells}

Wenjia Liu, Liqiang Zhang, Kun Xuan, Chenghu Hu, Shiyu Liu, Li Liao, Bei Li, Fang Jin, Songtao Shi and Yan Jin (D)

Bone Research (2020)8:29

; https://doi.org/10.1038/s41413-020-00107-z

Correction to: Bone Research https://www.nature.com/articles/ s41413-018-0029-4, published online 11 September 2018

During a re-read of our article [1], previously published in Bone Research, we noticed that Fig. 3b (second panel of line 4), which presented LAP2 $\beta$ immunostaining of first-passage MSCs from $\mathrm{Alpl}^{{ }^{+/-}}$mice at 4 months, was consistent to Fig. 7a (fourth panel of line 2), which was presented the LAP2 $\beta$ immunostaining of HPP MSCs. All the authors agree to rectify this mistake by rearranging Fig. 7 below.

This correction does not affect the results or conclusions of the above paper. We apologize for this typographical error and any inconvenience caused.

\section{REFERENCE}

1. Liu, W. et al. Alpl prevents bone ageing sensitivity by specifically regulating senescence and differentiation in mesenchymal stem cells. Bone Res. 6, 27 (2018).

\begin{abstract}
Open Access This article is licensed under a Creative Commons Attribution 4.0 International License, which permits use, sharing, adaptation, distribution and reproduction in any medium or format, as long as you give appropriate credit to the original author(s) and the source, provide a link to the Creative Commons license, and indicate if changes were made. The images or other third party material in this article are included in the article's Creative Commons license, unless indicated otherwise in a credit line to the material. If material is not included in the article's Creative Commons license and your intended use is not permitted by statutory regulation or exceeds the permitted use, you will need to obtain permission directly from the copyright holder. To view a copy of this license, visit http://creativecommons. org/licenses/by/4.0/.
\end{abstract}

(c) The Author(s) 2020 


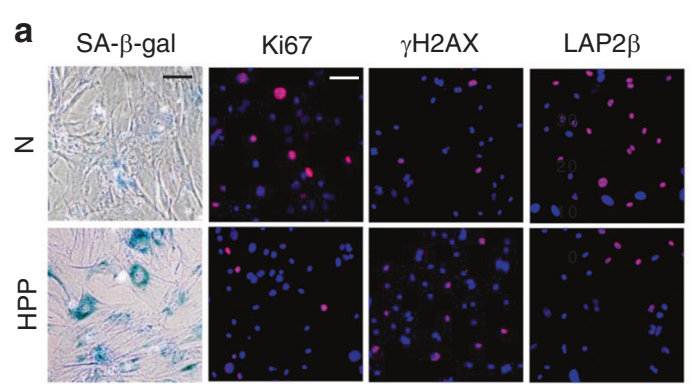

b

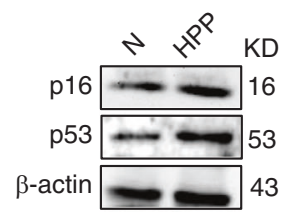

C
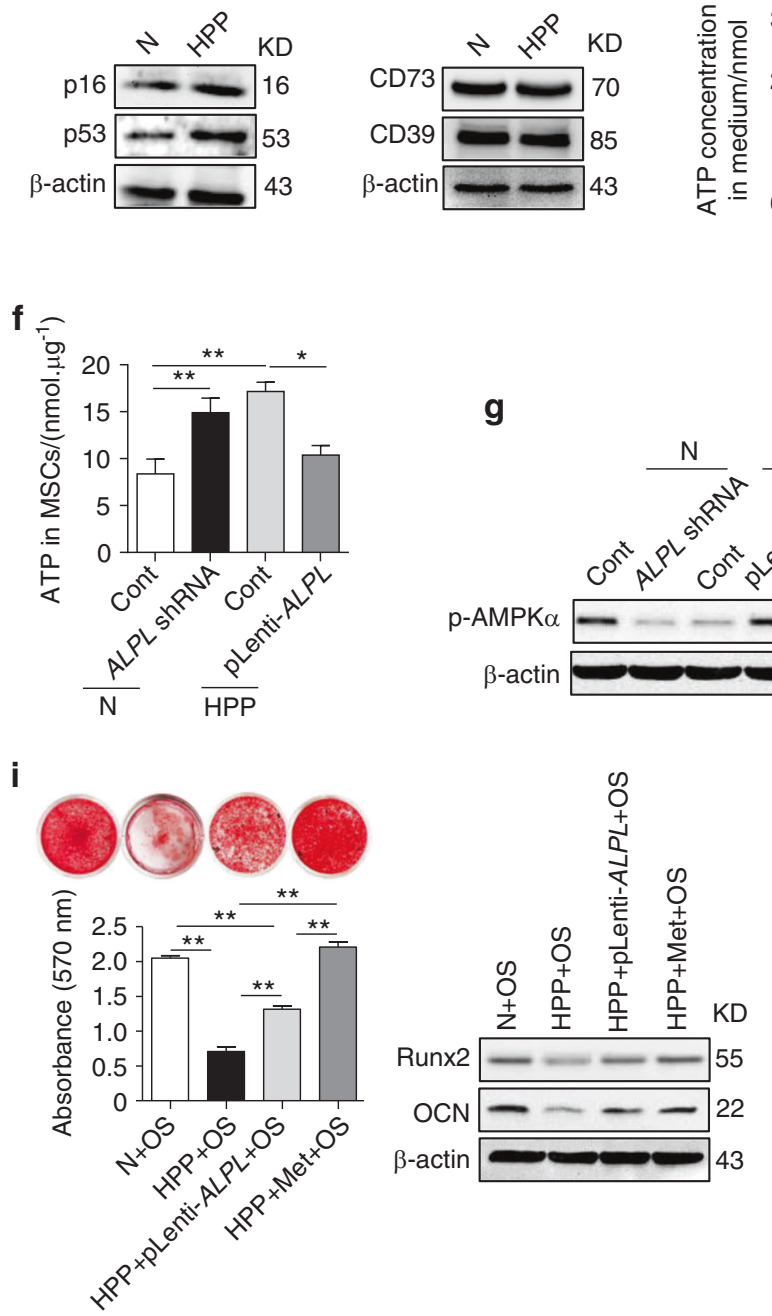

g

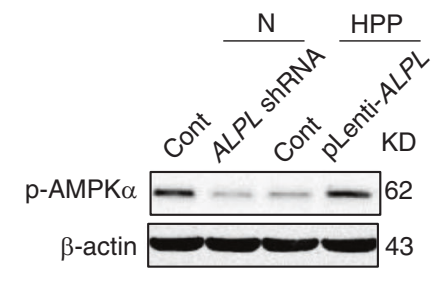

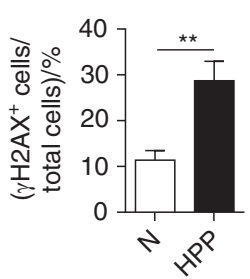

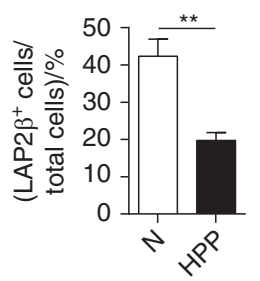

e

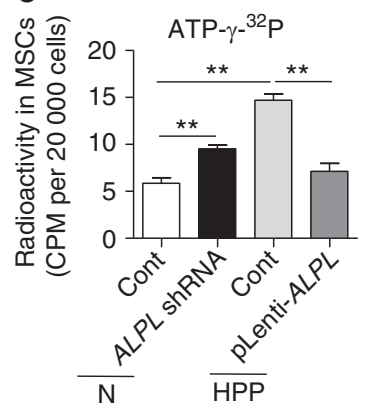

\section{d}

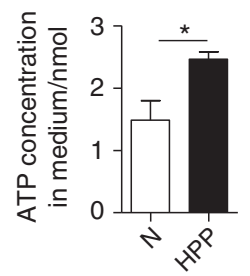

h
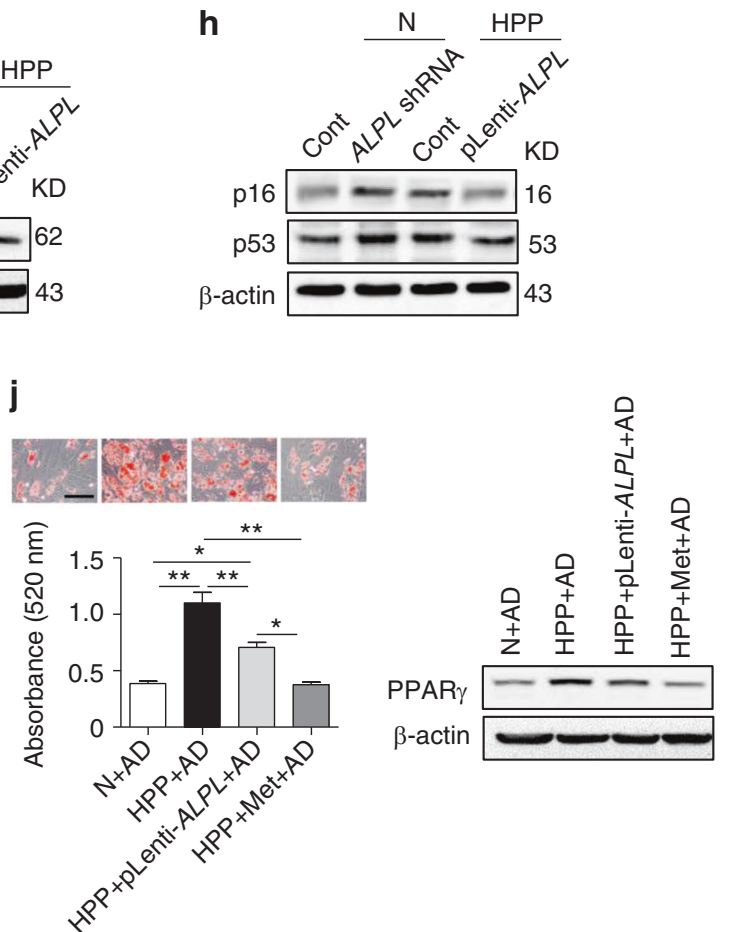

Fig. 7 Alpl also controls the differentiation and senescence of human MSCs via ATP-mediated inactivation of the AMPKa pathway. a SA$\beta$-gal staining and Ki67, $\gamma \mathrm{H} 2 \mathrm{AX}$ and LAP2 $\beta$ immunostaining of third-passage MSCs from normal controls and HPP patients. Quantification of $\mathrm{Ki} 7^{+}, \gamma \mathrm{H} 2 \mathrm{AX}{ }^{+}$and LAP2 $\beta^{+}$is indicated in the right panel. Scale bars: $50 \mu \mathrm{m}$. b Expression levels of ageing-specific genes in normal and HPP yiMSCs were examined by western blotting. Scale bars, $50 \mu \mathrm{m}$. c Expression levels of CD73 and CD39 in normal and HPP MSCs were examined by western blotting. d Extracellular ATP concentrations in normal and HPP MSC medium were examined by a regular ATP concentration assay. e Intracellular radioactivity was examined after a $1-\mathrm{h}$ treatment with ATP- $\gamma-{ }^{32} \mathrm{P}$ in different lentiviral vector transduction groups. $\mathrm{f}$ Intracellular ATP concentrations were assayed $48 \mathrm{~h}$ after the transduction of different lentiviral vectors. $\mathbf{g}$ Western blotting analysis of $\mathrm{p}$-AMPK $\alpha$ expression in normal control and the ALPL shRNA, HPP control and pLenti-ALPL groups. $\mathbf{h}$ Expression levels of p16 and p53 were assayed $48 \mathrm{~h}$ after the transduction of different lentiviral vectors. i HPP MSCs overexpressing ALPL or treatment with $0.1 \mathrm{mmol} \cdot \mathrm{L}^{-1}$ metformin, Alizarin Red staining and quantification of mineralized nodules were performed on day 28 after osteogenic induction (OS). Expression levels of Runx2 and OCN were examined by western blotting on day 7 after induction. $\mathbf{j}$ Oil Red $\mathrm{O}$ staining and quantification of fat depots were performed on day 14 after the adipogenic induction (AD). PPAR- $\gamma$ expression was examined on day 7 after induction by western blotting. Scale bars, $100 \mu \mathrm{m}$. (N) Normal control $n=5$, HPP (hypophosphatasia patient) $n=2$. The data are presented as the means \pm s.d. of each independent experiment performed in triplicate. ${ }^{*} P<0.05,{ }^{*} P<0.01$. e-f, $\mathbf{i}-\mathbf{j}$ One-way analysis of variance (ANOVA). a, $\mathbf{d}$ Unpaired two-tailed Student's t-test. 